\title{
Numerical simulation of diffusion-controlled droplet growth: Dynamical correlation effects
}

\author{
C. W. J. Beenakker* \\ Institute for Theoretical Physics, University of California, Santa Barbara, California 93106
}

(Received 24 December 1985)

\begin{abstract}
Diffusion-controlled coarsening (Ostwald ripening) of precipitated solutions is studied by numerical simulation. An algorithm is devised which exploits the screening of solute concentration fields, thereby removing the restriction to small systems of previous work. Simulation of the coarsening of 5000 droplets at $10 \%$ volume fraction reveals long-ranged dynamical correlations which broaden the droplet size-distribution function and increase the coarsening-rate coefficient.
\end{abstract}

An interesting and important development in studies of first-order phase transitions is the recent incorporation of dynamical correlation effects into the mean-field LifshitzSlyosov-Wagner (LSW) ${ }^{1,2}$ theory of diffusion-controlled droplet growth. The LSW theory deals with the final stage in precipitation from liquid or solid solutions, during which larger precipitated droplets grow at the expense of smaller ones, which disappear. The driving force for this process, known as coarsening or Ostwald ripening, is the increased solubility of the smaller droplets due to surface tension at the precipitate-solute interface. ${ }^{3}$ At very low volume fractions $\phi$ of the precipitate it suffices to consider the growth of a single droplet in an effective medium. This is the regime of validity of the LSW theory, in which correlations between positions and sizes of the droplets are neglected. The scaling laws obtained by LSW are well established: In the long-time limit the distribution function $P(a, t)$ of droplet radii $a$ at time $t$ takes the form $p(a / \bar{a}(t)) \bar{a}^{-4}(t)$, with the average radius $\bar{a}(t) \propto t^{1 / 3}$. However, the shape of the scaled distribution function $p(a / \bar{a})$ observed experimentally (even at rather low volume fractions) turns out to be considerably broader and less skewed than would follow from the LSW theory. An appealing explanation for this discrepancy, investigated very recently by Marder, ${ }^{4}$ is based on the effect of correlations which develop during coarsening. Because of the long range of diffusive interactions, these dynamical correlations may well play an important role even in dilute systems. Marder's theory is a perturbation expansion to first order in $\bar{a} / \lambda$, with $\lambda$ the screening length of diffusive interactions. Since $\lambda-\bar{a} / \phi^{1 / 2}$, this is an expansion to order $\phi^{1 / 2}$. Similar theories to this order have been studied by Marqusee and Ross, ${ }^{5}$ and by Tokuyama, Kawasaki and Enomoto, ${ }^{6,7}$ although not to the same extent.

In this paper I take an altogether different, nonperturbative approach, based on a direct numerical integration of the equations of motion. Such an approach (first proposed by Weins and $\mathrm{Cahn}^{8}$ ) has previously been taken by Voorhees and Glicksman (VG). ${ }^{9}$ Unfortunately, the systems studied by these authors were too small for dynamical correlations to develop during the simulation. The largest system consisted of 320 droplets initially; while this is already a rather low number for statistical purposes, it decreases even further as small droplets dissolve and disappear. To avoid early termination of the simulation, VG regularly added new droplets with the same size distribution-but at random locations, thereby destroying any dynamical correlations. The practical reason for the limitation to small systems of the VG study lies in the fact that the computation time required for their simulation increases very rapidly as $N^{3}$ with the number of droplets $N$. However, by exploiting the screening of diffusive interactions it is possible, as will be shown in this paper, to devise an algorithm for which the computation time and storage requirements increase only linearly with $N$. This will enable us to study much larger systems than considered previously, and to investigate the nature and effect of dynamical correlations in the coarsening process.

The starting point is the standard model of Ostwald ripening, ${ }^{3}$ in which the precipitate is assumed to consist of $N(t)$ immobile spherical particles [position vectors $\mathbf{R}_{\boldsymbol{i}}$, radii $\left.a_{i}(t)\right]$ with growth laws

$$
a_{i}^{2} \frac{d a_{i}}{d t}=Q_{i}, \quad i=1,2, \ldots, N .
$$

The growth rates $Q_{i}$ a determined from the quasistatic diffusion equation, with local-equilibrium boundary conditions on the surfaces of the droplets. The result can be written in the form

$$
Q_{i}=\alpha \sum_{J=1}^{N} Z_{i j}\left(\Delta-\frac{1}{a_{j}}\right)
$$

with $\alpha \equiv D d v c_{\infty}$ and $\Delta \equiv\left(c_{0}-c_{\infty}\right) / c_{\infty} d$. Here $D$ is the diffusion coefficient of the solute, $c_{\infty}$ the molar solute concentration in equilibrium with a flat interface, $v$ the molar volume of the precipitate, and $d$ a capillary length (typically of order $10^{-7} \mathrm{~cm}$ ). The constant $c_{0}$ denotes a reservoir solute concentration representing the boundary condition "at infinity." The transport matrix $Z$ satisfies the sum rule $^{10} \sum_{i} Z_{i j}=\Sigma_{j} Z_{i j}=0$, valid in the limit of an infinite system. Note that because of this identity the evolution of the system is independent of $\Delta$, reflecting screening of the bulk of the system from the reservoir. Furthermore, it follows that

$$
\sum_{j} Q_{j}=0
$$

and hence that the volume fraction $\phi \equiv \Sigma_{j} \frac{4}{3} \pi a_{j}^{3} N / V$ (with $V$ the volume of the system) is conserved. ${ }^{10,11}$ In the monopolar approximation, Eq. (2) takes the form

$$
\frac{1}{a_{i}} Q_{i}+\sum_{\substack{j \\ j \neq i}} \frac{1}{R_{i j}} Q_{j}=\alpha\left(\Delta-\frac{1}{a_{i}}\right), \quad i=1,2, \ldots, N,
$$

with $R_{y} \equiv\left\|R_{j}-R_{i}\right\|$. As shown in Ref. 12, use of the monopolar approximation limits the validity of the theory to 
the regime $\phi \leq 0.1$.

The VG simulation is based on Eqs. (1), (3), and (4) above. The most time-consuming step in their simulation is the calculation of the growth rates, which is computationally of order $N^{3}$. In their scheme the interactions of each droplet $i$ with every other droplet $j$ are accounted for. This is to a certain extent a waste, since the major contributions to the growth rate of a droplet come from neighbors which are at most a few screening lengths away. By exploiting the physical phenomenon of screening, the full problem can be approximated (to a sufficient accuracy, as we shall see) by one which is only of order $N$ computationally. The outline of this numerical scheme is as follows. For a given droplet $i_{0}$ we determine a cluster of neighbors $C\left(i_{0}\right)$ within a certain range. Equation (3) plus the set of Eqs. (4) with $i \in C\left(i_{0}\right)$ is then solved for $Q_{i_{0}}$, with the proviso that the sum over $j$ in these equations is also restricted to $j \in C\left(i_{0}\right)$. In this way the growth rate of the central droplet $i_{0}$ is determined with neglect of all couplings between the cluster of neighbors and other droplets in the system. This procedure is repeated for each $i_{0}=1,2, \ldots, N$, whereafter the growth laws (1) are integrated over one time step. Note the special role which $\Delta$ plays in this scheme: In the full problem this quantity (the reservoir supersaturation) is an irrelevant parameter, as discussed above. Upon truncation of the interaction range, however, $\Delta$ becomes an additional degree of freedom to be determined by Eq. (3), which is then an independent equation. There is of course nothing sacred about this procedure, and one can think of alternative ways of fixing $\Delta$, but this particular choice turns out to give a fairly rapid convergence with increasing interaction range.

Carrying out the above algorithm on a VAX-11/750 com- puter, we have simulated the coarsening of a system with initially 5000 droplets at $\phi=0.1$. This volume fraction is sufficiently high for the screening length to be of the order of the interdroplet separation, but not so high that dipolar contributions to the interactions can no longer be neglected. ${ }^{12}$ The droplets are located in a cube with periodic boundary conditions, to minimize the effect of a finite system size. Each cluster of interacting neighbors contains at least 11 droplets and has a minimal radius of $3 \lambda_{\text {loc }}$, where $\lambda_{\text {loc }} \equiv\left(4 \pi \bar{a}_{\text {loc }} \bar{n}_{\text {loc }}\right)^{-1 / 2}$ is the local screening length calculated from the average radius $\bar{a}_{\mathrm{loc}}$ and number density $\bar{n}_{\mathrm{loc}}$ of the droplets in that particular cluster. This local criterion gives some flexibility in dealing with different environments in the system. To integrate the growth laws (1) we use Adams' fourth-order predictor-corrector formulas, with one corrector cycle. The time step chosen is $10^{-2} \tau_{C}$ (with $\tau_{C}=\bar{a}^{3} / D v c_{\infty} d$ the coarsening time scale) and increases, therefore, as the average radius grows. When the radius of a droplet reaches $10^{-2} \vec{a}$ it is removed from the system. Clusters are updated after every three time steps. Initially the droplet-size-distribution function was a narrow Gaussian, and the droplets were located at random positions (no overlaps). At the end of this first run (when the number of droplets had dropped to about 300) the system was well into the scaling regime and its scaled size-distribution function seemed to have reached a steady state. To be more certain that this was indeed a time-independent state, I then performed a second run, starting afresh with 5000 droplets which now had the distribution of sizes found at the end of the previous run. In addition, this new system reproduced ${ }^{13}$ the two correlation functions $\chi_{00}(r)$ and $\chi_{11}(r)$, which are moments of the pair distribution function,

$$
\chi_{n m}\left(\left\|\mathbf{R}^{\prime}-\mathbf{R}\right\|\right)=\left(\frac{N}{V}\right)^{-2} \int_{0}^{\infty} d a \int_{0}^{\infty} d a^{\prime}\left(\frac{a}{\bar{a}}-1\right)^{n}\left(\frac{a^{\prime}}{\bar{a}}-1\right)^{m} P_{2}\left(a, \mathbf{R} ; a^{\prime}, \mathbf{R}^{\prime}\right)
$$

Here $P_{2}\left(a, \mathbf{R} ; a^{\prime}, \mathbf{R}^{\prime}\right)$ is the probability of finding both a droplet of radius $a$ at $\mathbf{R}$ and a droplet of radius $a^{\prime}$ at $\mathbf{R}^{\prime}$. The results of this second run are shown in Figs. 1-5.

The most questionable aspect of this approach seems to us to be the truncation of the interaction range. The approximate nature of this device manifests itself clearly in a spurious drift of the precipitate volume fraction, which during the simulation increases from 0.10 to 0.11 . To assess the consequences of this approximation, a comparison was made of the results of two runs $A$ and $B$ in which different sized clusters were used: (A) a run with minimal cluster radius $2.5 \lambda_{l o c}$ and on average 12 droplets per cluster; (B) the run described above, with minimal cluster radius $3 \lambda_{10 c}$ and 19 droplets per cluster, on average. Both runs started from the same droplet configuration. The increase in interaction range substantially improved the volume fraction drift, which decreased from $20 \%$ in run $\mathrm{A}$ to $10 \%$ in run B. The evolution of the droplet-size-distribution function, however, was essentially unaffected, as can be seen from Figs. 1 and 2 where the results of both runs are plotted. The conclusion is that the effects of the truncation are reasonably well under control.

Now let us turn to a discussion of the results. As shown in Figs. 1 and 2, the system has entered what appears to be a steady state, in which the width $\sigma \equiv\left\langle(a / \bar{a}-1)^{2}\right\rangle^{1 / 2}$ and skewness $\kappa \equiv\left\langle(a / \bar{a}-1)^{3}\right\rangle / \sigma^{3}$ (with $\langle\cdots\rangle$ denoting the average) of the scaled size-distribution function are time independent, apart from statistical fluctuations. The average radius follows the growth law $\bar{a}(t)=\bar{a}(0)\left(1+K t / \tau_{C}\right)^{1 / 3}$, with $\tau_{C}=\bar{a}^{3}(0) / D v c_{\infty} d$ and $K=0.88 \pm 0.03$. The scaled droplet-radius distribution function $p(a / \bar{a})$ (normalized to

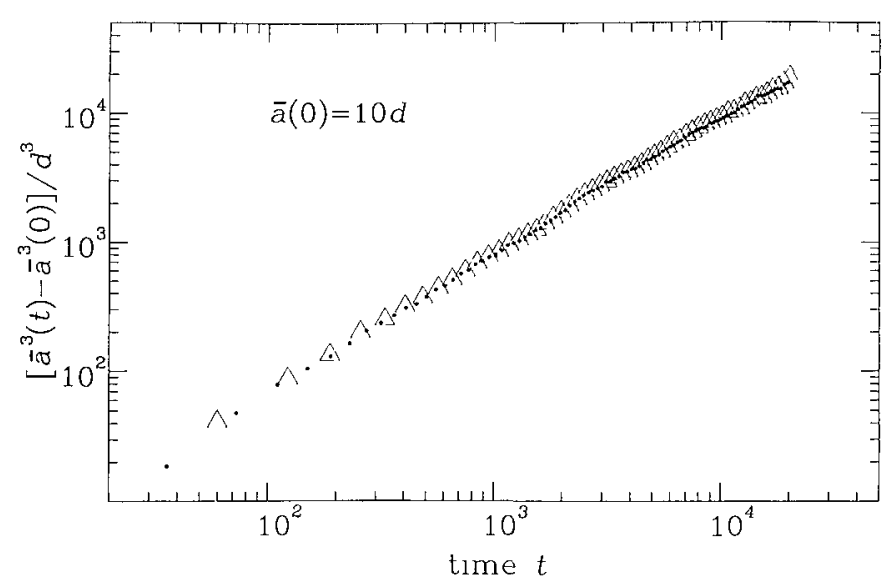

FIG. 1. Time dependence of the average droplet radius. Triangles and dots represent two runs with different cluster sizes (referred to in the text as runs $A$ and $B$, respectively). Time in units of $d^{2} / D v c_{\infty}$. 


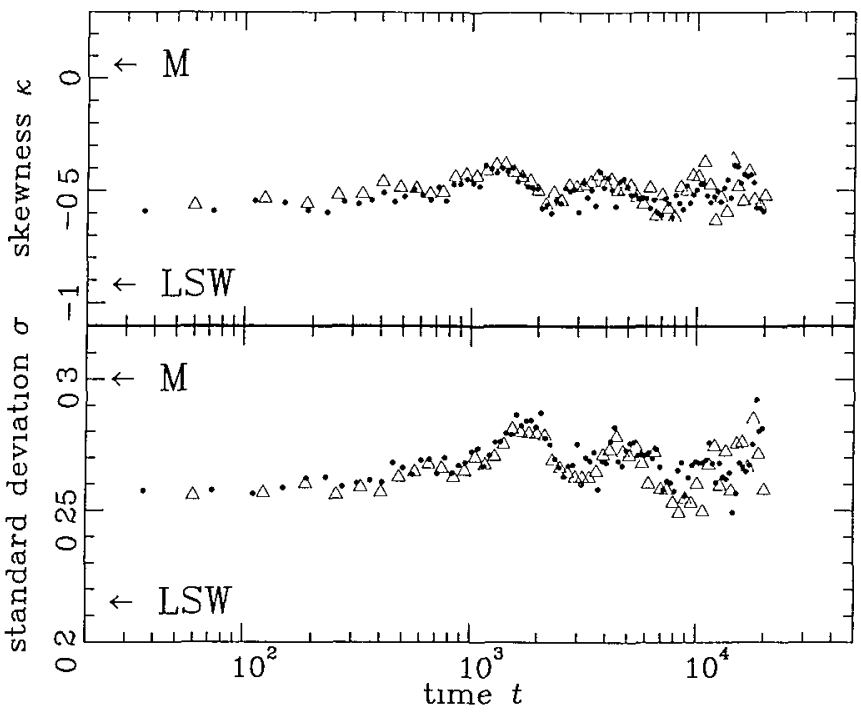

FIG 2 Time dependence of the width and skewness of the scaled droplet-radius distribution function Triangles and dots as in Fig 1 Arrows indicate the theoretical results of Marder (M) and LSW Time in units of $d^{2} / D v c_{\infty}$

unity) is shown in Fig 3 The distribution of sizes found is broader and less skewed than the mean-field LSW result-but not at ail so broad and symmetric as follows from Marder's theory for the effect of correlations (Marder's curve was actually computed for $\phi=005$, his result at $\phi=01$ is still slightly broader ${ }^{14}$ ) Also shown in Fig 3 is the distribution function resulting from the VG simulation, which is less broad than the result obtained here and resembles more closely the LSW function The coarsening rate coefficient $K$ shows a similar trend We find $K=088$, which is much larger than the LSW value of 044 -but smaller than Marder's result of 112 VG find $K=069 \pm 003$, which again lies between my result and the

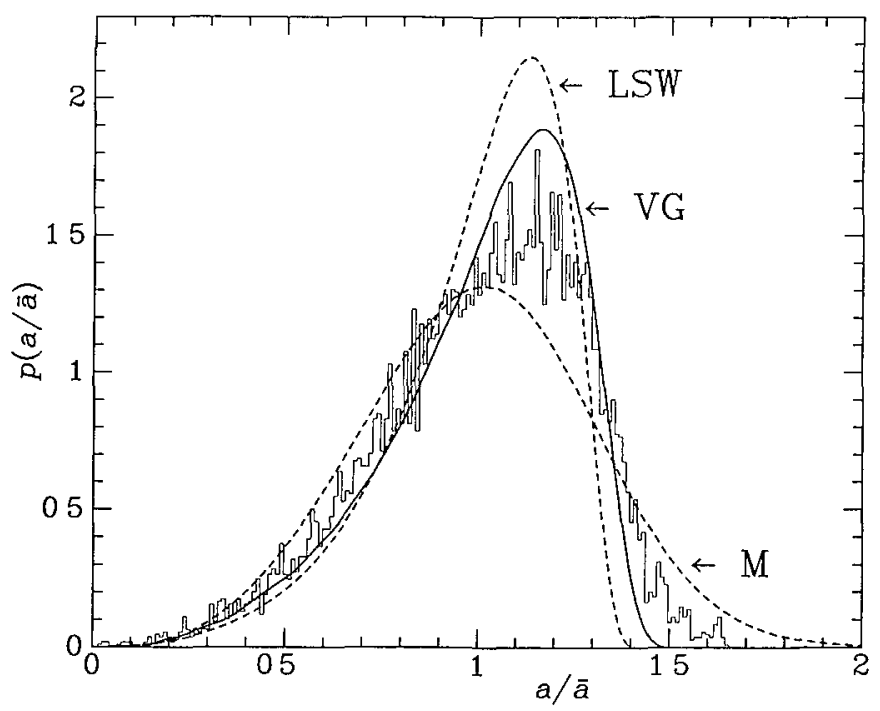

FIG 3 Scaled distribution of droplet radı (normalized to unity) The histogram is calculated from 25 distributions corresponding to the final 25 dots in $F_{1 g} 2$ The solid curve results from the VG simulation, dashed curves are the theoretical results of Marder and LSW

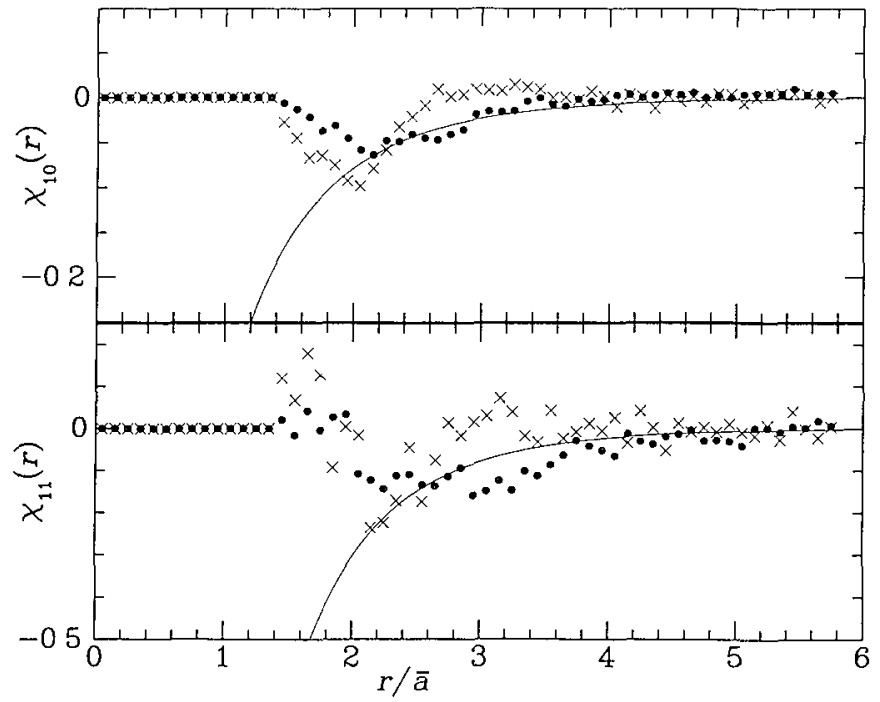

FIG 4 Correlation functions $\chi_{10}$ and $\chi_{11}$, defined in Eq (5) Dots the present simulation, solid curve Marder's theory For comparison, the results for a hard-sphere distribution are also shown (crosses)

\section{LSW theory}

One may attribute the finding of stronger departures from the LSW theory than observed in the VG simulation to the fact that dynamical correlations were destroyed in the sımulation In Fig 4 we have plotted two correlation functions $\chi_{11}(r)$ and $\chi_{10}(1)$, defined in Eq (5) The dots follow from my simulation, whereas the crosses show the same functions for a hard-sphere distribution of the droplets These latter correlations would have been present in the VG simulation during which the system was regularly "reshuffled" Although the sign and order of magnitude of each correlation function does not differ in the two cases, the dynamical correlations are of considerably longer range than the hard-sphere correlations, which are due purely to excluded volume effects ${ }^{15}$ This is consistent with the picture of correlations developing as a result of diffusive interactions, which (at the volume fraction considered) extend over several droplet radı The general long-range form of

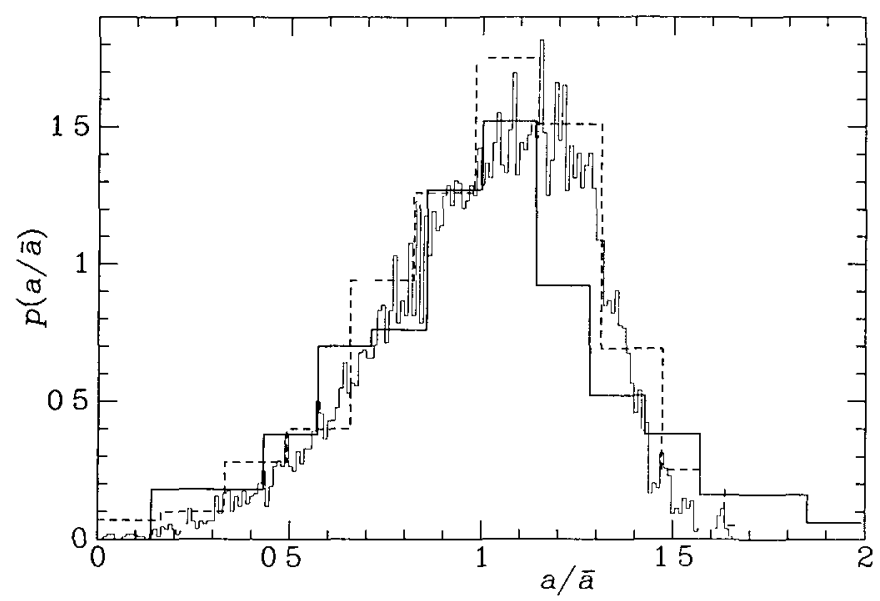

FIG 5 Comparison of the distribution of droplet radis found here (cf Fig 3) with experimental histograms taken from Ref 16 (dashed lines) and Ref 17 (solid lines) 
the correlation functions agrees also quite well with the results of Marder's perturbation theory (the solid curves in Fig. 4). At shorter separations, however, Marder's functions diverge as $1 / r$, rather than falling off to zero as they should. This breakdown of the perturbation theory at short-length scales is most likely responsible for the excessive broadening of the droplet-size-distribution function obtained by Marder at this volume fraction. Presumably this deficiency will be less important at lower densities. Figure 5 , finally, shows the distribution of sizes obtained here together with data from two experiments at $\phi=0.1 .^{16,17}$ The experimental histograms are clearly broader than the LSW result, and are in reasonable agreement with the simulation. More accurate experiments are needed, however, before one can conclude that the broadening is fully explained by correlation effects neglected in the LSW theory. It would be particularly interesting and important to have experimental data for such correlation functions as discussed in this paper.

The author has benefitted greatly from discussions with J. S. Langer, M. Marder, and J. Reiter. Financial support from the Niels Stensen Stichting is gratefully acknowledged. This research was supported in part by the U.S. Department of Energy under Grant No. DE-FG03-84ER45108 and by the National Science Foundation under Grant No. PHY8217853, supplemented by funds from the National Aeronautics and Space Administration, at the University of California at Santa Barbara.
"Present address: Philips Research Laboratories, NL-5600 JA Eindhoven, The Netherlands.

11. M. Lifshitz and V. V. Slyosov, J. Phys. Chem. Solids 19, 35 (1961).

${ }^{2}$ C. Wagner, Z. Elektrochem. 65, 581 (1961).

${ }^{3}$ For a recent review, see P. W. Voorhees, J. Stat. Phys. 38, 231 (1985).

${ }^{4}$ M. Marder, Phys. Rev. Lett. 55, 2953 (1985).

5J. A. Marqusee and J. Ross, J. Chem. Phys. 80, 536 (1984).

${ }^{6}$ M. Tokuyama and K. Kawasaki, Physica A 123, 386 (1984).

${ }^{7}$ M. Tokuyama, K. Kawasaki, and Y. Enomoto, Physica A 134, 323 (1986).

${ }^{8} \mathrm{~J}$. J. Weins and J. W. Cahn, in Sintering and Related Phenomena, edited by G. C. Kuczynski (Plenum, New York, 1973).

${ }^{9}$ P. W. Voorhees and M. E. Glicksman, Acta Metall. 32, 2001 (1984); 32, 2013 (1984).

${ }^{10}$ C. W. J. Beenakker and J. Ross, J. Chem. Phys. 83, 4710 (1985).

${ }^{11}$ Constancy of the volume fraction is within the present scheme a consequence of the quasistatic approximation to the diffusion equation. The actual volume fraction increase in the coarsening process is of order $v c_{\infty} d / \bar{a}$, which is negligible in the late stage of the process.

${ }^{12}$ C. W. J. Beenakker and J. Ross, J. Chem Phys. 84, 3857 (1986).

${ }^{13}$ The method used to reproduce the correlation functions, due to Marder, is a potential algorithm. To reproduce $\chi_{00}$, for example, we start with an arbitrary distribution of points. A random move of one point is then attempted, which is accepted if it decreases the $L^{2}$ separation of the correlation function to the function which we seek to reproduce. Convergence is rapid, within $10 \mathrm{~N}$ attempted moves.

${ }^{14} \mathrm{M}$. Marder (private communication).

${ }^{15}$ The simulation algorithm does not completely prevent droplets from overlapping as they grow. Because of the strong interactions between two droplets at short separations overlaps are, however, rather unlikely. In fact, we found that the actual fraction of the volume occupied by the precipitate was less than $\frac{4}{3} \pi \sum_{i} a_{i}{ }^{3} / V$ by not more than one part in $10^{4}$.

${ }^{16}$ A. J. Ardeil and R. B. Nicholson, J. Phys. Chem. Solids 27, 1793 (1966).

${ }^{17}$ M. Chaturvedi and D. W. Chung, J. Inst. Met. 101, 253 (1973). 